\title{
Respiratory illness and respiratory syncytial virus hospitalization in infants with a tracheostomy following prophylaxis with palivizumab
}

\author{
Bosco Paes $^{1}$ (D) $\cdot$ Mahwesh Saleem ${ }^{2} \cdot$ Doyoung Kim $^{2} \cdot$ Krista L. Lanctôt $^{2} \cdot$ lan Mitchell $^{3}$
}

Received: 8 April 2019 / Accepted: 13 May 2019 / Published online: 22 May 2019

(C) Springer-Verlag GmbH Germany, part of Springer Nature 2019

\begin{abstract}
Data on respiratory-related illness and respiratory syncytial virus (RSV) infection in children with a tracheostomy are sparse. We determined respiratory illness hospitalization (RIH) and RSV-related hospitalization (RSVH) hazard ratios in children with a tracheostomy following prophylaxis compared with infants' prophylaxed for standard indications (prematurity $\leq 35$ weeks' gestational age, bronchopulmonary dysplasia, and significant congenital heart disease) and children with complex medical disorders. Children who received $\geq 1$ injection of palivizumab were prospectively enrolled across 32 Canadian sites during the RSV season. Respiratory illness event data were collected monthly. Data were analyzed using $t$ tests, chi-square tests, and Cox proportional hazards adjusted for confounders. A total of 23,597 infants were enrolled; 220 tracheostomy, 19,402 standard indications, 3975 complex medical disorders. Of the 220 tracheostomy infants, 30 had bronchopulmonary dysplasia, 18 were premature, 12 had congenital heart disease, and 160 had other medical complexities. RIH and RSVH incidences (tracheostomy, standard indications, complex medical disorders) were $24.5 \%, 6.2 \%$, and $9.8 \%$ and $2.0 \%, 1.5 \%$, and $1.8 \%$ respectively. RIH hazard was significantly higher in tracheostomy infants compared with standard indications (HR $=1.8,95 \%$ CI $1.1-3.0, p=0.02$ ) but was similar between the tracheostomy and complex medical disorders groups (HR $=1.3,95 \% \mathrm{CI} 0.7-2.2, p=0.37)$. RSVH hazard was also similar in tracheostomy infants relative to standard indications and complex medical disorders (both $p>0.75$ ). Children with tracheostomies who received palivizumab had an increased RIH hazard compared with the standard indications group. Similar RSVH hazard between tracheostomy, standard indications, and complex medical disorders groups suggests that children with tracheostomies may benefit from palivizumab by reducing RSVH during the RSV season.
\end{abstract}

Keywords Hospitalization $\cdot$ Palivizumab $\cdot$ Respiratory illness $\cdot$ Respiratory syncytial virus $\cdot$ Tracheostomy

\section{Introduction}

Children with a tracheostomy comprise a diverse population that spans those who require the procedure to resolve upper

Bosco Paes

paes@mcmaster.ca

1 Department of Pediatrics (Neonatal Division) - HSC-3A, McMaster University, 1280 Main Street West, Hamilton, Ontario L8S 4K1, Canada

2 Department of Medical Outcomes and Research in Economics (MORE®) Research Group, Sunnybrook Health Sciences Centre, Toronto, Ontario, Canada

3 Department of Pediatrics, Alberta Children's Hospital, University of Calgary, Calgary, Alberta, Canada airway obstruction primarily due to congenital anomalies to those who require respiratory support for long-term chronic respiratory and neuromuscular disease. In a recent review, the largest percentage of children with a tracheostomy was aged $<1$ year (range 4-78\%) with a statistically significant increase in the respective cohort from 41.3 to $63 \%$ between 1985-1994 and 1995-2004, respectively [1]. Over the last 30 years, several investigators have reported an evolution in the indications for a tracheostomy in children from principally infectious causes to predominantly those who require respiratory support for neurologic impairment and traumatic injury [2-5].

Complication rates resulting from pediatric tracheostomy vary by time, namely intra, early, and late postoperative and the inclusion or exclusion of serious events such as air leak syndrome, erosive wound infection, tube obstruction, accidental decannulation, respiratory arrest, and death $[1,3,5-7]$. 
Overall, adverse event rates range between 10 and $63 \%$ among published reports and the frequency of complications is dependent on the study duration and severity of illness [3, 6-8]. Similarly, mortality rates in the pediatric population are influenced by age at the time of death, the underlying medical condition governing the indication for tracheostomy (range 2$59 \%$ ) and direct association with the procedure (range 0 $5.9 \%)$ [1-3, 6, 9-14]. Important predictors of mortality are prematurity, young age, cardiopulmonary and acquired neurologic disease, cancer, and children with medical complexity $[1,2,9-14]$.

The literature regarding the contribution of respiratoryrelated illness, especially respiratory syncytial virus (RSV) to the morbidity incurred by children with a tracheostomy is extremely sparse and not clearly defined. Schweiger et al. briefly reported that among 123 children with a tracheostomy, 38 (31\%) died. Pneumonia of undefined etiology and clinical complications resulted in the death of 11 children [6]. Yaneza et al. documented that respiratory tract infection occurred in $35.6 \%(n=85 / 239)$ outpatient visits among children with a tracheostomy, but none of the patients were hospitalized [5]. Furthermore, the majority of the focus in regard to lower respiratory tract infections in children with a tracheostomy is on pathogenic bacteria $[15,16]$.

RSV is the main viral organism responsible for lower respiratory tract illness in children $<2$ years of age worldwide and is associated with significant morbidity and mortality especially in children with complex medical conditions $[17,18]$. In randomized clinical trials, palivizumab, a humanized monoclonal antibody, has been proven to be safe and effective in reducing RSV-related hospitalization (RSVH) in premature children and those with bronchopulmonary dysplasia and congenital heart disease $[19,20]$. The objective of our study was to determine respiratory illness hospitalization (RIH) and RSVH hazard ratios in children with a tracheostomy compared with children prophylaxed for standard indications (SI; prematurity $\leq 35$ weeks gestational age, bronchopulmonary dysplasia, and hemodynamically significant congenital heart disease) and complex medical disorders (CMD) in the Canadian RSV Evaluation Study of Palivizumab (CARESS).

\section{Materials and methods}

CARESS is a prospective, observational registry of children who received $\geq 1$ dose of palivizumab from 2005 to 2017 in 32 Canadian sites [21]. The study was reviewed and approved independently, by each site's respective research ethics board. Informed consent was obtained from parents or legal guardians prior to patient enrollment. Baseline data were collected at the time of recruitment on patient demographics, prior medical history, neonatal course, and details of palivizumab administration. Interviews were subsequently conducted at monthly time intervals with the subjects' primary caregivers during the respective RSV season. At each interview information regarding palivizumab administration, changes in baseline data, hospitalization for respiratory illness, diagnostic tests for RSV-related infection, reasons for discontinuation of prophylaxis, and incurred adverse events were recorded. In the event of a hospitalization, the relevant hospital records of the subject were reviewed by the site's research nurse for the indication for hospitalization, length of stay (regular ward versus intensive care), days on respiratory support and/or intubation, RSV diagnostic test results, and clinical diagnosis, as documented in the discharge summary.

\section{Subjects, definitions, and outcomes}

Any child who received $\geq 1$ injection of palivizumab during the respective RSV season was eligible and was enrolled at the start of the RSV season following the first dose of palivizumab and preferably before the third injection, in order to secure sequential data collection and analysis. Children were excluded if they were receiving any other similar monoclonal antibody within a clinical trial, or if their parents or caregivers were unable to communicate in English or French.

All children with a tracheostomy, aged $<2$ years, were eligible irrespective of the indication and underlying medical condition. The SI group comprised children currently approved for RSV prophylaxis by the majority of international consensus guidelines (premature infants $\leq 35$ completed weeks gestational age, children with bronchopulmonary dysplasia, or hemodynamically significant congenital heart disease) [22-25]. Children with a CMD included those with serious medical conditions such as airway anomalies, pulmonary disorders, neuromuscular impairment, and immunodeficiency who were approved for off-label prophylaxis provincially on a case-by-case basis [26-28].

Adherence with the monthly protocol of injections was defined as an infant who received $\geq 5$ or at least the expected number of palivizumab injections within the recommended time intervals between injections, during the respective RSV season [29]. Dosing intervals for palivizumab were defined as 16 to 35 days between the first and second injection and $30 \pm$ 5 days for subsequent injections [29-31]. Children hospitalized with a symptomatic lower respiratory tract infection during the RSV season were tested for the presence of RSV infection. RSV positivity was confirmed by enzyme or immunofluorescent assay, real-time PCR or a positive viral culture from a posterior nasopharyngeal swab, wash or tracheal secretions. RIH was defined as all patients admitted with a respiratory tract illness that had both positive and negative RSV test results. The primary endpoints of this study were the hazard for first RIH and RSVH across the groups (tracheostomy, SI, and $\mathrm{CMD}$ ). 


\section{Statistical analysis}

Data were analyzed by standard descriptive methods, comparative statistics (chi-square, analysis of variance (ANOVA), and Student's $t$ tests), and regression analysis using IBM SPSS Statistics 20 (IBM, Armonk, NY). Demographic comparisons between the tracheostomy, SI, and CMD groups were performed using ANOVA for continuous variables and chisquare tests for categorical variables. A $p$ value $<0.05$ was considered statistically significant.

RIH and RSVH rates were calculated for descriptive purposes. The RIH rate was determined by the number of children hospitalized for a respiratory illness divided by the total number of children. The RSVH rate was calculated as the incidence of RIH, multiplied by the number of RSV-positive subjects, and divided by the number of patients tested for RSV. Cox proportional hazards analyses were conducted to compare RIH and RSVH risks across groups and were adjusted for potential confounders (demographic and neonatal variables that were significantly different between the groups $[p<0.05]$ ) that correlated to time to hospitalization using a backward conditional method. A sensitivity analysis was also conducted excluding all patients in the SI category (prematurity, bronchopulmonary dysplasia, and significant congenital heart disease) and those with pulmonary hypertension. This was performed to control for any known risk factors that enhance the risk for RSVH and to estimate the true risk of RIH or RSVH in the subjects with a tracheostomy.

\section{Results}

A total of 23,597 children were included between the 2005 and 2016 RSV seasons (n); tracheostomy (220), SI (19402), and CMD (3975). Of the 220 children with a tracheostomy who received prophylaxis in the SI group, 18 were premature, 30 had bronchopulmonary dysplasia, and 12 had significant congenital heart disease. One-hundred and sixty children with a tracheostomy had CMD.

Across the groups, children received on average, $4.4 \pm$ 1.3 palivizumab injections per RSV season and 58.6\% with a tracheostomy were perfectly adherent to the protocol (received $\geq 5$ injections or at least the expected number of palivizumab injections within the recommended time intervals between injections per season) compared with $63.5 \%$ and $68.3 \%$ of the SI and CMD groups respectively. The overall perfect adherence rate among the three groups was $64.2 \%$. The demographic and neonatal characteristics are shown in Tables 1 and 2. All the variables were significantly different between the study groups $(p<0.05)$. A greater proportion of tracheostomy infants were male, Caucasian, and had a family history of atopy compared with the SI and CMD groups. During the neonatal course, a higher proportion of children with a tracheostomy required respiratory support, oxygen therapy, and had surgery for patent ductus arteriosus and documented necrotizing enterocolitis and sepsis, than the SI and CMD groups. Children with a tracheostomy were older and bigger at enrollment and required longer durations of hospital stay, respiratory support, and oxygen therapy than the other sub-populations. Respiratory illness and RSV-related hospitalizations are described in Table 3. A total of 1655 children were hospitalized for 1970 respiratory-related events. Of the 220 subjects with a tracheostomy, 54 were hospitalized for a total of 74 respiratory illness events. 19.1\% (316/1655) hospitalized children were RSV-positive; 4 (tracheostomy), 250 (SI), and 62 (CMD).

The characteristics of RSVHs among the recruited subjects are shown in Table 4. All 4 RSVHs in the children with a tracheostomy, who received prophylaxis, occurred in the second year of life. Of the RSV-positive children, there were significant group differences in the proportion who were admitted with apnea (tracheostomy, 25.0\%; SI, 16.4\%; and CMD, $4.8 \%\left[\chi^{2}=6.5, p=0.038\right]$ ) and bronchiolitis (tracheostomy, 50.0\%; SI, 77.6\%; and CMD, 69.4\% $\left[\chi^{2}=6.3, p=\right.$ 0.042]) (Table 4). A higher proportion of RSV-positive children with a tracheostomy $(25.0 \%)$ were hospitalized with respiratory arrest, compared with the SI (1.6\%) and CMD groups $(3.2 \%)\left[\chi^{2}=10.1, p=0.006\right]$ (Table 4).

Cox proportional hazard analyses were adjusted for potential confounders as shown in Tables 1 and 2. The hazard plot for RIH is shown in Fig. 1. The RIH hazard of children with a tracheostomy was significantly higher compared with the SI group (HR $=1.8,95 \%$ CI 1.1-3.0, $p=0.022)$. The RIH hazard was similar between the tracheostomy and CMD groups $(\mathrm{HR}=1.3,95 \%$ CI 0.7-2.2, $p=0.369$ ). No significant difference in RSVH hazard was observed in children with a tracheostomy relative to the SI and CMD groups (tracheostomy versus SI: $\mathrm{HR}=1.2,95 \%$ CI 0.4-3.6, $p=0.803$; tracheostomy versus CMD: HR= $1.2,95 \%$ CI $0.4-3.9, p=0.751$ ).

A sub-analysis of the tracheostomy group was conducted to determine whether there were differences in the risk of RIH and RSVH after excluding children with established risk factors for RSVH (prematurity, bronchopulmonary dysplasia and congenital heart disease $[n=60]$, and those with pulmonary hypertension $[n=7]$ ). The analysis was performed controlling for neonatal respiratory support, oxygen therapy, documented sepsis, enrollment age and weight, and gestational age. Tracheostomy subjects with risk factors $(n=67)$ had similar RIH and RSVH hazards relative to those without risk factors $(n=153)$ (RIH: $\mathrm{HR}=1.3,95 \%$ CI $0.7-2.5, p=$ 0.361 ; RSVH: $\mathrm{HR}=8.2,95 \%$ CI $0.5-130.2, p=0.136$ ). 
Table 1 Demographic characteristics of the enrolled subjects

Demographic characteristics

\begin{tabular}{|c|c|c|c|c|c|}
\hline & $\operatorname{Trach}(n=220)$ & SI $(n=19,402)$ & $\mathrm{CMD}(n=3975)$ & $X^{2}$ or $H$ & $p$ value \\
\hline Male, $n(\%)$ & $138(62.7)$ & $10,996(56.7)$ & $2164(54.6)$ & 9.8 & 0.008 \\
\hline Caucasian, $n(\%)$ & $120(74.5)$ & $269(68.0)$ & $13,916(72.0)$ & 42.5 & $<0.0005$ \\
\hline Aboriginal, $n(\%)$ & $7(3.2)$ & $796(4.1)$ & $123(3.1)$ & 0.2 & 0.010 \\
\hline Daycare attendance, $n(\%)$ & $4(1.8)$ & $531(2.7)$ & $395(10.0)$ & 455.4 & $<0.0005$ \\
\hline Multiple birth, $n(\%)$ & $26(11.8)$ & $5870(30.3)$ & $640(16.1)$ & 358.0 & $<0.0005$ \\
\hline Exposure to smoking, $n(\%)$ & $55(25.0)$ & $5732(29.5)$ & $968(24.4)$ & 45.0 & $<0.0005$ \\
\hline Siblings, $n(\%)$ & $143(65.0)$ & $12,261(63.2)$ & $2601(65.4)$ & 7.4 & 0.026 \\
\hline Siblings in daycare or school-aged, $n(\%)$ & $76(49.4)$ & $5715(44.6)$ & $1328(51.0)$ & 36.6 & $<0.0005$ \\
\hline$\geq 5$ people in the household, $n(\%)$ & $43(19.5)$ & $4860(25.0)$ & $842(21.2)$ & 29.6 & $<0.0005$ \\
\hline Family history of atopy, $n(\%)$ & $99(45.4)$ & $7702(39.8)$ & $1688(42.8)$ & 13.9 & 0.001 \\
\hline Median enrollment age (months [IQR]) & 13.7 [6.9-21.9] & $3.2[1.5-5.8]$ & $7.6[3.1-14.2]$ & 1812.8 & $<0.0005$ \\
\hline Median gestational age (week [IQR]) & $36.2[31.3-38.1]$ & $31.9[29.1-34.1]$ & $38.0[35.0-39.1]$ & 3665.4 & $<0.0005$ \\
\hline Median enrollment weight (g [IQR]) & $8405.0[6335.0-10,257.5]$ & $4020.0[2820.0-6042.0]$ & $7140.0[4900.0-9250.0]$ & 2826.6 & $<0.0005$ \\
\hline
\end{tabular}

$C M D$, complex medical disorders; $I Q R$, interquartile range; $S I$, standard indications; Trach, tracheostomy

All variables were significantly different between the groups $(p<0.05)$ and adjusted for in subsequent analyses

\section{Discussion}

Published reports on respiratory tract infections with specific etiology causing hospitalization in children with a tracheostomy have been limited [5, 6]. More recently, Shay et al. [32] noted that upper respiratory disease featured among the top ten admission diagnosis in children with a pediatric tracheotomy $(n=14 / 373 ; 3.8 \%)$ among which $43(11.5 \%)$ had pneumonia and $43(11.4 \%)$ were classified as other lower respiratory disease but the pathogenic organisms were not reported. Akangire et al. [33] conducted a retrospective cohort study on 110 infants aged $<2$ years with a tracheostomy who were ventilator dependent. Tracheostomies were performed principally for bronchopulmonary dysplasia (28\%) and other acquired chronic pulmonary disease $(23 \%)$. The main cause for re-hospitalization in the total cohort who received RSV prophylaxis was viral-related respiratory tract infections (26\%). Rhinovirus/enterovirus was the most common virus isolated (37\%), followed by RSV (9\%). Tan et al. [34] reviewed 90 children from 2002 to 2016 with a tracheostomy among which $42(46.7 \%)$ were hospitalized in a tertiary care center in Taiwan with 141 episodes of respiratory tract infections. Of this cohort, $125(88.7 \%)$ had definite or possible bacterial pneumonia and $12(8.5 \%)$ were classified as nonbacterial pneumonia, during a median follow-up of 3.15 years. A total of 20 viral episodes were identified among the cases of non-bacterial pneumonia, at a median age of 1.24 years, and the majority $(n=12 ; 60 \%)$ were due to RSV. The median age

Table 2 Neonatal characteristics of the enrolled subjects

Neonatal characteristics

\begin{tabular}{|c|c|c|c|c|c|}
\hline & $\operatorname{Trach}(n=220)$ & SI $(n=19,402)$ & $\operatorname{CMD}(n=3975)$ & $X^{2}$ or $F$ & $p$ value \\
\hline Days of neonatal stay (mean $\pm \mathrm{SD}$ ) & $157.9( \pm 149.6)$ & $49.8( \pm 65.1)$ & $40.2( \pm 71.4)$ & 283.1 & $<0.0005$ \\
\hline Respiratory support, $n(\%)$ & $180(81.8)$ & $12,342(63.6)$ & 1535 (38.6) & 901.7 & $<0.0005$ \\
\hline Duration in days (mean $\pm \mathrm{SD}$ ) & $112.2( \pm 136.9)$ & $23.0( \pm 32.1)$ & $24.3( \pm 49.6)$ & 494.7 & $<0.0005$ \\
\hline Oxygen therapy, $n(\%)$ & $161(73.2)$ & $9913(51.1)$ & $1640(41.3)$ & 176.9 & $<0.0005$ \\
\hline Duration in days (mean $\pm \mathrm{SD}$ ) & $113.4( \pm 143.0)$ & $34.8( \pm 60.6)$ & $28.4( \pm 55.9)$ & 132.6 & $<0.0005$ \\
\hline Documented necrotizing enterocolitis & $11(5.0)$ & $617(3.2)$ & $77(1.9)$ & 20.7 & $<0.0005$ \\
\hline Surgery for patent ductus Arteriosus, $n(\%)$ & $21(9.5)$ & $1000(5.2)$ & $95(2.4)$ & 67.4 & $<0.0005$ \\
\hline Documented sepsis, $n(\%)$ & $70(32.1)$ & $2702(14.0)$ & $411(10.3)$ & 102.3 & $<0.0005$ \\
\hline
\end{tabular}

$C M D$, complex medical disorders; SI, standard indications; Trach, tracheostomy

All variables were significantly different between the groups $(p<0.05)$ and adjusted for in subsequent analyses 
Table 3 Unadjusted RIH and RSVH incidences

\begin{tabular}{lllll}
\hline & Trach $(n=220)$ & SI $(n=19,042)$ & CMD $(n=3975)$ & Total $(n=23,597)$ \\
\hline RIH & 54 & 1212 & 389 & 1655 \\
RIH incidence & $24.5 \%$ & $6.2 \%$ & $9.8 \%$ & $7.0 \%$ \\
Tested for RSV & 49 & 1025 & 337 & 1411 \\
RSVH & 4 & 250 & 62 & 316 \\
RSVH incidence & $2.0 \%$ & $1.5 \%$ & $1.8 \%$ & $1.6 \%$ \\
\hline
\end{tabular}

$C M D$, complex medical disorders; $R I H$, respiratory illness-related hospitalization; $R S V$, respiratory syncytial virus; $R S V H$, respiratory syncytial virus-related hospitalization; SI, standard indications; Trach, tracheostomy for tracheostomy was 3.05 months and the median duration from tracheostomy to infection was 0.57 years. Cerebral palsy and gastroesophageal reflux disease were independently associated with the occurrence of respiratory tract infections. In our study, the mean gestational age of the subjects was 34.7 weeks and enrollment for RSV prophylaxis occurred at a mean chronological age of 16.2 months. All of the children who underwent a tracheostomy had a more complex neonatal course with a 3.6-fold longer duration of hospital stay and higher morbidities in terms of oxygen and respiratory support requirement, sepsis, necrotizing enterocolitis, and surgical intervention for patent ductus arteriosus. The higher proportional morbidity in the children with a tracheostomy compared with the SI and CMD groups and the accompanying prematurity place them at greater risk for RSVH.

The overall RIH incidence in the Taiwan study [34] was $46.7 \%$ and the RSVH incidence of presumed untreated patients was $8.8 \%(12 / 137)$ compared with $24.5 \%$ and $2 \%$ respectively in the prophylaxed subjects in the CARESS study.
Since the demographics of the underlying conditions that necessitated a tracheostomy were similar to our study and the rest of the published, scientific literature, RSV prophylaxis during the RSV season (November to March in temperate climates) may possibly achieve an approximately $77 \%$ reduction in RSVH, but this needs to be validated in further prospective, well-designed clinical trials. Akangire. et al. reported an $\mathrm{RSVH}$ rate of $4 \%$ in a smaller sample size of infants with a tracheostomy who received palivizumab $(n=100)$ but adherence with the protocol was not delineated [33]. This would result in a corresponding reduction in RSVH of $54.5 \%$, compared with $77 \%$ in our study but the former finding is similar to the overall reductions in RSVH reported in the randomized, placebo controlled IMpact study (55\%) and the cardiac controlled trial $(45 \%)$.

It is difficult to extrapolate the precise morbidities experienced by patients following RSVH in the Tan et al. [34] study since the data were broadly grouped under the umbrella of non-bacterial pneumonia. Based on the latter
Table 4 Characteristics of respiratory syncytial virus hospitalizations among the recruited subjects (total $n=316$ RSV hospitalizations)

\begin{tabular}{llllll}
\hline$n=$ Number of RSV hospitalizations & $\begin{array}{l}\text { Trach } \\
(n=4)\end{array}$ & $\begin{array}{l}\text { SI } \\
(n=250)\end{array}$ & $\begin{array}{l}\text { CMD } \\
(n=62)\end{array}$ & $\begin{array}{l}X^{2} \text { or } \\
F\end{array}$ & $\begin{array}{l}p \\
\text { value }\end{array}$ \\
\hline Apnea, $n(\%)$ & $1(25.0)$ & $41(16.4)$ & $3(4.8)$ & 6.5 & 0.038 \\
Bronchiolitis, $n(\%)$ & $2(50.0)$ & $194(77.6)$ & $43(69.4)$ & 6.3 & 0.042 \\
Decreased oxygen saturation, $n(\%)$ & $3(75.0)$ & $106(44.9)$ & $34(54.8)$ & 2.6 & 0.268 \\
Inability to maintain oral intake, $n(\%)$ & $1(25.0)$ & $106(42.4)$ & $29(46.8)$ & 0.6 & 0.744 \\
Pneumonia, $n(\%)$ & $1(25.0)$ & $48(19.2)$ & $21(33.9)$ & 4.8 & 0.090 \\
Respiratory arrest, $n(\%)$ & $1(25.0)$ & $4(1.6)$ & $2(3.2)$ & 10.1 & 0.006 \\
Respiratory distress, $n(\%)$ & $3(75.0)$ & $170(68.0)$ & $52(83.9)$ & 2.6 & 0.274 \\
Respiratory support required, $n(\%)$ & $1(25.0)$ & $83(33.2)$ & $25(40.3)$ & 0.7 & 0.705 \\
Days on respiratory support (mean $\pm \mathrm{SD})$ & $0.3 \pm 0.6$ & $2.6 \pm 6.8$ & $2.0 \pm 3.8$ & 0.4 & 0.679 \\
Length of hospital stay in days (mean \pm & $5.0 \pm 5.5$ & $8.2 \pm 11.0$ & $7.1 \pm 5.6$ & 0.5 & 0.626 \\
$\quad$ SD) & $2(50.0)$ & $71(28.4)$ & $15(24.2)$ & 1.8 & 0.404 \\
ICU admission, $n(\%)$ & $1.5 \pm 2.4$ & $2.5 \pm 7.0$ & $1.4 \pm 3.4$ & 0.8 & 0.450 \\
ICU length of stay in days (mean $\pm \mathrm{SD})$ & $1(25.0)$ & $30(12.0)$ & $7(11.3)$ & 0.7 & 0.692 \\
Intubation required, $n(\%)$ & $0.3 \pm 0.5$ & $1.4 \pm 6.1$ & $0.7 \pm 2.7$ & 0.5 & 0.621 \\
Days on intubation (mean \pm SD) & & & &
\end{tabular}

$d f=2 ; p<0.05$ is statistically significant

$C M D$, complex medical disorders; $I C U$, intensive care unit; $R S V$, respiratory syncytial virus; $S I$, standard indications; Trach, tracheostomy 


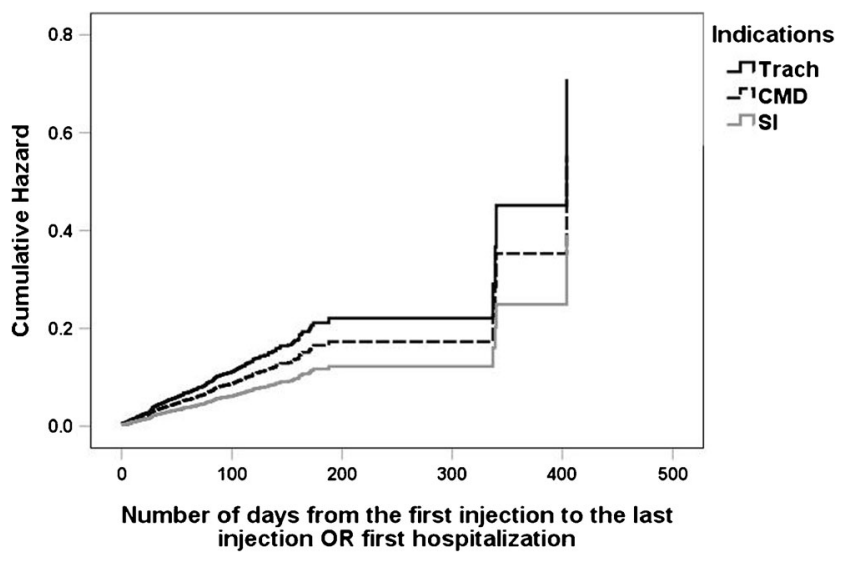

Fig. 1 Cox proportional hazard analysis of respiratory-illness hospitalization. RIH hazard of children with a tracheostomy was significantly higher compared with SI (HR $=1.8,95 \%$ CI 1.1-3.0, $p=0.022)$. RIH hazard was similar between the tracheostomy and CMD groups $(\mathrm{HR}=$ $1.3,95 \%$ CI 0.7-2.2, $p=0.369$ ). CMD, complex medical disorders; RIH, respiratory illness hospitalization; SI, standard indications; Trach, tracheostomy

categorization, three patients $(25 \%)$ required ventilator support; four $(25 \%)$ were admitted to intensive care and the total duration of hospital stay was $5.67 \pm 2.55$ days. In our study, the children with a tracheostomy who received prophylaxis and were hospitalized with RSV were more commonly admitted with apnea. Of note, a significantly higher proportion presented with respiratory arrest $(25 \%$, $p=0.006$ ). Morbidities incurred during the hospital course included higher but statistically insignificant admission to intensive care and intubation compared with the SI and CMD groups. The overall hazard for RIH was significantly higher in the children with tracheostomies compared with those who received prophylaxis for standard, approved indications and the RIH rate (24\%) aligns with the rate reported by Akangire et al. [33] but was much lower than the almost $50 \%$ RIH rate documented by Tan et al. [34]. The RSVH rate in the tracheostomy group in this study was similar to the SI and CMD group suggesting that prophylaxis with palivizumab may have dampened the severity of RSV infection and corresponding related hospitalization.

It is important to emphasize that at the time of RSVH, all the children with tracheostomies enrolled in the CARESS study were symptomatic. With regard to bacterial infections, there are no established, evidence-based guidelines for the diagnosis of respiratory infections in children with tracheostomies. Multicenter studies indicate significant variation in the approach to diagnosis, care, and outcomes of these children following hospitalization [15, 16, 35-37]. Drysdale et al. [38] in a recent report on the interpretation of viral tests in children indicated that the detection of a virus especially by PCR in association with respiratory disease progression signifies that the virus is the cause of clinical disease. In our study, real-time
PCR has evolved as the diagnostic test of choice in recent years and has superseded other tests for RSV and other viral detection when indicated.

This is the largest, prospective, comprehensive study of RIH and RSVH in children with tracheostomies who received prophylaxis during the RSV season over a period of 11 years. The prospective design and close surveillance of the subjects for specific outcomes of interest across 32 sites affords generalizability across the population in Canada and is applicable to similar cohorts in other countries worldwide. The association of tracheostomies with apnea and respiratory arrest, albeit a small sample size due to the positive treatment effect of palivizumab, should raise awareness that when these children present with bronchiolitis and lower respiratory tract infection in the emergency room, local causes such as accidental decannulation and pre-existing pathology should first be excluded. A rapid screen for RSV should be performed and hospitalization should be considered for a child who is RSVpositive and clinically symptomatic.

There are several limitations that merit consideration. First, the absence of a control arm restricts an estimate of the true efficacy and benefit of palivizumab in the reduction of RSVH in this population. Second, although the majority of subjects with a tracheostomy were evaluated for RSV following hospitalization (91\%), the absence of testing in 5 children, and the range of diagnostic tests conducted with varying sensitivity and specificity, may have resulted in an underestimate of the incidence of RSVH [38]. Third, the registry focuses on RSVrelated illness following hospitalization, and data on other viral pathogens causing respiratory tract illness were not systematically collected. Fourth, children were enrolled in the CARESS registry at different time points which may have influenced the RSVH rate relative to the use of prophylaxis. However, this was accounted for by the Cox proportional analysis of either the number of days from the first to the last injection or time to first RIH and RSVH. There was a lack of homogeneity across the groups but the differences were adjusted in the Cox proportional hazards analysis. The heterogeneity illustrates real-world evidence of children with medical complexity who receive palivizumab. Of note, children with a tracheostomy are not indifferent to those with CMDs. Last, the sample size of the tracheostomy group was relatively small compared with the SI and CMD groups but is consistent with other published studies $[3,5,6]$. This may have compromised our ability to detect statistically significant differences in morbidities following RSVH between the groups.

\section{Conclusion}

This study involves the largest cohort of children with a tracheostomy who have received RSV prophylaxis. Children enrolled in the CARESS registry, who received 
palivizumab during the RSV season, had an increased RIH hazard compared with those who received prophylaxis for SI. The finding of a similar RSVH hazard between the tracheostomy, SI, and CMD groups suggests that infants with a tracheostomy, independent of documented risk factors for RSVH, may benefit from palivizumab by reducing RSVH and attendant morbidities during the RSV season.

Acknowledgments The authors would like to thank the following investigators in the CARESS 2005-2017 seasons: Dr. Candice Bjornson and Dr. Ian Mitchell (Alberta Children's Hospital), Dr. Mark Chilvers (BC Children's Hospital), Dr. Georges Caouette [Centre Hospitalier de l'Université (CHU) Laval], Dr. Marc Lebel (CHU Sainte-Justine), Dr. Mario Eddy Dumas (CHU Sherbrooke), Dr. Charles Hui (Children's Hospital of Eastern Ontario), Dr. Ann Bayliss (Credit Valley Hospital), Dr. Bruno DiGravio (Grand River Hospital), Dr. Jean-Pierre Doray (Hôpital Charles LeMoyne), Dr. Dora Stinson (IWK Health Centre), Dr. Apostolos Papageorgiou (Jewish General Hospital), Dr. Marianna Mitchell (Lakeridge Health Oshawa), Dr. David Lee and Dr. April Price (London Health Sciences Centre), Dr. Aaron Chiu (Manitoba Institute of Child Health), Dr. Bosco Paes (McMaster Children's Hospital), Dr. Roderick Canning (Moncton Hospital), Dr. Anne-Marie Canakis and Dr. Jesse Papenburg (Montreal Children's Hospital), Dr. Karel O'Brien (Mount Sinai Hospital), Dr. Karen Chang (Rouge Valley Hospital), Dr. Koravangattu Sankaran (Royal University Hospital), Dr. Vincent Ho (Royal Victoria Hospital), Dr. Larry Chang (Southlake Regional Health Centre), Dr. Cecil Ojah (St. John Regional Hospital), Dr. Sanja Avdic (St Joseph's Health Centre), Dr. Upton Allen (Sick Kids Hospital), Dr. Carina Majaesic (Stollery Children's Hospital), Dr. Marc Blayney (Sudbury Regional Hospital), Dr. Brian Simmons Sunnybrook Health Sciences Centre), Mr. Kiang Tang and Dr. Jelena Popovic (Toronto East General Hospital), Dr. Frank Jagdis (Victoria General Hospital), Dr. Ivor Margolis (William Osler Health Centre), and Dr. Godfrey Bacheyie (Windsor Regional Hospital).

We thank the patients and families across Canada who participated in the project.

Role of authors BP, MS, DK, KL, IM, BP: conception and design, analysis and interpretation of data, drafting of article;

MS, DK, KL, BP: analysis and interpretation of data;

All: reviewed manuscript and facilitated revisions;

All: final approval of manuscript and assume public responsibility for the content.

Source of funding The CARESS registry is funded by an investigatorinitiated grant from AbbVie Corporation, Canada. The CARESS registry is registered under ClinicalTrials.gov; Identifier: NCT00420966.

\section{Compliance with ethical standards}

Conflict of interest B.P. I.M. and K.L. have received investigatorinitiated research funding or received compensation as advisors or lecturers from AbbVie Corporation and MedImmune. M.S. and D.K. have no conflicts of interest to declare. AbbVie had no role in the study design, data collection, analysis and interpretation, decision to publish, or preparation of the manuscript. No honorarium, grant, or other form of payment was given to anyone to produce the manuscript.

Ethical approval The study was approved by The Research Ethics Board of each of the 32 centers involved in the recruitment of subjects.

\section{References}

1. Dal'Astra AP, Quirino AV, Caixêta JA, Avelino MA (2017) Tracheostomy in childhood: review of the literature on complications and mortality over the last three decades. Braz J Otorhinolaryngol 83(2):207-214

2. McPherson ML, Shekerdemian L, Goldsworthy M, Minard CG, Nelson CS, Stein F, Graf JM (2017) A decade of pediatric tracheostomies: indications, outcomes, and long-term prognosis. Pediatr Pulmonol 52(7):946-953

3. Ogilvie LN, Kozak JK, Chiu S, Adderley RJ, Kozak FK (2014) Changes in pediatric tracheostomy 1982-2011: a Canadian tertiary children's hospital review (2014). J Pediatr Surg 49(11):1549-1553

4. Gergin O, Adil EA, Kawai K, Watters K, Moritz E, Rahbar R (2016) Indications of pediatric tracheostomy over the last 30 years: has anything changed? Int J Pediatr Otorhinolaryngol 87:144-147

5. Yaneza MM, James HL, Davies P, Harrison S, McAlorum L, Clement WA, Kubba H (2015) Changing indications for paediatric tracheostomy and the role of a multidisciplinary tracheostomy clinic. J Laryngol Otol 129(12):882-886

6. Schweiger C, Manica D, Becker CF, Abreu LSP, Manzini M, Sekine L, Kuhl G (2017) Tracheostomy in children: a ten-year experience from a tertiary center in southern Brazil. Braz J Otorhinolaryngol 83(6):627-632

7. Watters KF (2017) Tracheostomy in infants and children. Respir Care 62(6):799-825

8. Campisi P, Forte V (2016) Pediatric tracheostomy. Semin Pediatr Surg 25(3):191-195

9. Funamura JL, Yuen S, Kawai K, Gergin O, Adil E, Rahbar R, Watters K (2017) Characterizing mortality in pediatric tracheostomy patients. Laryngoscope 127(7):1701-1706

10. Watters K, O’Neill M, Zhu H, Graham RJ, Hall M, Berry J (2016) Two-year mortality, complications, and healthcare use in children with medicaid following tracheostomy. Laryngoscope 126(11): 2611-2617

11. Ilçe Z, Celayir S, Tekand GT, Murat NS, Erdoğan E, Yeker D (2002) Tracheostomy in childhood: 20 years experience from a pediatric surgery clinic. Pediatr Int 44(3):306-309

12. Maxwell BG, McMillan KN (2014) Tracheostomy in children with congenital heart disease: a national analysis of the kids' inpatient database. PeerJ 2:e568

13. Wood D, McShane P, Davis P (2012) Tracheostomy in children admitted to paediatric intensive care. Arch Dis Child 97(10):866869

14. Mahida JB, Asti L, Boss EF, Shah RK, Deans KJ, Minneci PC, Jatana KR (2016) Tracheostomy placement in children younger than 2 years: 30-day outcomes using the National Surgical Quality Improvement Program Pediatric. JAMA Otolaryngol Head Neck Surg 142(3):241-246

15. Russell CJ, Mack WJ, Schrager SM, Wu S (2017) Care variations and outcomes for children hospitalized with bacterial tracheostomyassociated respiratory infections. Hosp Pediatr 7(1):16-23

16. Sanders CD, Guimbellot JS, Muhlebach MS, Lin FC, Gilligan P, Esther CR Jr (2018) Tracheostomy in children: epidemiology and clinical outcomes. Pediatr Pulmonol 53(9):1269-1275

17. Bont L, Checchia PA, Fauroux B, Figueras-Aloy J, Manzoni P, Paes B, Simões EA, Carbonell-Estrany X (2016) Defining the epidemiology and burden of severe respiratory syncytial virus infection among infants and children in western countries. Infect Dis Ther 5(3):271-298

18. Scheltema NM, Gentile A, Lucion F, Nokes DJ, Munywoki PK, Madhi SA, Groome MJ, Cohen C, Moyes J, Thorburn K et al (2017) Global respiratory syncytial virus-associated mortality in young children (RSV GOLD): a retrospective case series. Lancet Glob Health 5(10):e984-e991 
19. The IMpact-RSV Study Group (1998) Palivizumab, a humanized respiratory syncytial virus mono- clonal antibody, reduces hospitalization from respiratory syncytial virus infection in high-risk infants. Pediatrics 102(3 Pt 1):531-537

20. Feltes TF, Cabalka AK, Meissner HC, Piazza FM, Carlin DA, Top FH Jr, Connor EM, Sondheimer HM, Cardiac Synagis Study Group (2003) Palivizumab prophylaxis reduces hospitalization due to respiratory syncytial virus in young children with hemodynamically significant congenital heart disease. J Pediatr 143(4):532-540

21. Paes B, Mitchell I, Li A, Harimoto T, Lanctôt K (2013) Respiratoryrelated hospitalizations following prophylaxis in the Canadian registry for palivizumab (2005-2012) compared to other international registries. Clin Dev Immunol 2013:917068

22. Bollani L, Baraldi E, Chirico G, Dotta A, Lanari M, Del Vecchio A, Manzoni P, Boldrini A, Paolillo P, Di Fabio S, Orfeo L, Stronati M, Romagnoli C, Italian Society of Neonatology (2015) Italian Society of Neonatology revised recommendations concerning palivizumab prophylaxis for respiratory syncytial virus (RSV). Ital J Pediatr 41: 97

23. Figueras Aloy J, Carbonell Estrany X (2015) Comité de Estándares de la SENeo. Update of recommendations on the use of palivizumab as prophylaxis in RSV infections. An Pediatr (Barc) 82(3):199.e1-199.e2

24. Robinson JL, Le Saux N (2015) Canadian Paediatric Society ID, Immunization Committee. Preventing hospitalizations for respiratory syncytial virus infection. Paediatr Child Health 20(6):321-326

25. American Academy of Pediatrics Committee on Infectious Diseases; American Academy of Pediatrics Bronchiolitis Guidelines Committee (2014) Updated guidance for palivizumab prophylaxis among infants and young children at increased risk of hospitalization for respiratory syncytial virus infection. Pediatrics 134(2):e620-e638

26. Manzoni P, Paes B, Lanctôt KL, Dall'Agnola A, Mitchell I, Calabrese S, Maule M, Girardi E, Harimoto T, Li A (2017) Outcomes of infants receiving palivizumab prophylaxis for respiratory syncytial virus in Canada and Italy: an international, prospective cohort study. Pediatr Infect Dis J 36(1):2-8

27. Paes B, Mitchell I, Li A, Lanctôt KL (2012) Respiratory hospitalizations and respiratory syncytial virus prophylaxis in special populations. Eur J Pediatr 171(5):833-841

28. Gaboli M, de la Cruz ÒA, de Agüero MI, Moreno-Galdó A, Pérez GP, de Querol MS (2014) Use of palivizumab in infants and young children with severe respiratory disease: a Delphi study. Pediatr Pulmonol 49(5):490-502
29. Chan P, Li A, Paes B, Abraha H, Mitchell I, Lanctôt KL, CARESS investigators (2015) Adherence to palivizumab for respiratory syncytial virus prevention in the Canadian registry of palivizumab. Pediatr Infect Dis J 34(12):e290-e297

30. Wong SK, Li A, Lanctôt KL, Paes B (2018) Adherence and outcomes: a systematic review of palivizumab utilization. Expert Rev Respir Med 12(1):27-42

31. Robbie GJ, Zhao L, Mondick J, Losonsky G, Roskos LK (2012) Population pharmacokinetics of palivizumab, a humanized antirespiratory syncytial virus monoclonal antibody, in adults and children. Antimicrob Agents Chemother 56(10):4927-4936

32. Shay S, Shapiro NL, Bhattacharyya N (2018) Revisits after pediatric tracheotomy: airway concerns result in returns. Int J Pediatr Otorhinolaryngol 104:5-9

33. Akangire G, Manimtim W, Nyp M, Townley N, Dai H, Norberg M, Taylor JB (2017) Factors leading to rehospitalization for tracheostomized and ventilator-dependent infants through 2 years of age. J Perinatol 37(7):857-863

34. Tan CY, Chiu NC, Lee KS, Chi H, Huang FY, Huang DT, Chang L, Kung YH, Huang CY $(2018,2018)$ Respiratory tract infections in children with tracheostomy. J Microbiol Immunol Infect. https:// doi.org/10.1016/j.jmii.2018.07.002

35. Zhu H, Das P, Roberson DW, Jang J, Skinner ML, Paine M, Yuan J, Berry J (2015) Hospitalizations in children with preexisting tracheostomy: a national perspective. Laryngoscope 125(2):462-468

36. Rusakow LS, Guarín M, Wegner CB, Rice TB, Mischler EH (1998) Suspected respiratory tract infection in the tracheostomized child: the pediatric pulmonologist's approach. Chest 113(6):1549-1554

37. Cline JM, Woods CR, Ervin SE, Rubin BK, Kirse DJ (2012) Surveillance tracheal aspirate cultures do not reliably predict bacteria cultured at the time of an acute respiratory infection in children with tracheostomy tubes. Chest 141(3):625-631

38. Drysdale SB, Kelly DF (2018) How to use respiratory viral studies. Arch Dis Child Educ Pract Ed. https://doi.org/10.1136/ archdischild-2016-311858

Publisher's note Springer Nature remains neutral with regard to jurisdictional claims in published maps and institutional affiliations. 